Original Article

\title{
A comparison of the shoulder and trunk muscle activity according to the various resistance condition during push up plus in four point kneeling
}

\author{
Jung-Bin Kim, PT, MSc1), Chang-Kyo Yun, PT, MSc1)*, Myoung-hyo Lee, PT, PhD²) \\ 1) Department of Physical Therapy, College of Rehabilitation Science, Graduate School, Daegu \\ University: 15 Jilyang, Gyeongsan-si, Kyeongbuk 712-714, Republic of Korea \\ 2) Department of Physical Therapy, Daegu Fatima Hospital, Republic of Korea
}

\begin{abstract}
Purpose] This study was to compare the shoulder and trunk muscle activity according to the various resistance condition during knee push-up-plus. [Subjects and Methods] Twenty healthy adults participated in this study (9 males and 11 females). The subjects performed dominant-leg-extended knee push-up-plus apply to resistance in the direction of abduction, adduction, extension and the flexion. The surface Electromyography activities of the upper trapezius, serratus anterior, homolateral external oblique and the heterolateral internal oblique were measured. The Electromyography activities of each muscle were compared using a one-way repeated analysis of variance. [Results] The Electromyography activities of serratus anterior and external oblique muscles between the resistance directions were significantly increased extension. The Electromyography activities of heterolateral internal oblique muscle between the resistance directions were significantly increased adduction. [Conclusion] To suggest use of the decision exercise tolerance orientation when muscle strengthening exercises for shoulder and trunk according to variation resistance in lower extremity during Push-up-plus. Key words: Serratus anterior muscle, Knee push up plus, Resistance direction
\end{abstract}

(This article was submitted Aug. 10, 2016, and was accepted Sep. 20, 2016)

\section{INTRODUCTION}

High-quality functions of the scapulae depend on capabilities of the scapular muscles, which affect both static or dynamic stability and control of movement of the scapulae ${ }^{1)}$. According to the research, the weakened serratus anterior muscle may induce scapular winging due to lack of maintaining maximal length tension of the muscle, excessive muscle activity can be shown in the upper trapezius for compensation of the winging, and increase in winging may induce abnormal movements in the scapulae to cause the scapular impingement ${ }^{2,3)}$. According to Mottram those who have the connective tissues impeding functional movements of the scapulae and imbalance of force couple in muscles should recover the normal position through isometric exercise and training which provide stability ${ }^{4}$. Ellenbecker and Davies recommended push-up plus exercise as an effective closed-kinetic chain exercise on selective training of the serratus anterior muscle and for the muscles helpful for stabilization of the scapulae ${ }^{5)}$.

Recent researches show that stabilization of the scapulae is associated with other body segments; stabilization of the scapulae forms significant kinetic chain due to its connection from the trunk to the upper limbs, promoting several patterns of muscle activation with which the force from the feet on the ground is delivered to the hands ${ }^{6,7)}$. There have been many researches on effects of push-up plus exercise on the activity of muscles stabilizing the scapulae ${ }^{8)}$, but few researchers

*Corresponding author. Chang-kyo Yun (E-mail: puhaha1116@naver.com)

(C2017 The Society of Physical Therapy Science. Published by IPEC Inc.

This is an open-access article distributed under the terms of the Creative Commons Attribution Non-Commercial No Derivatives (by-nc-nd) License $<$ http://creativecommons.org/licenses/by-nc-nd/4.0/>. 
Table 1. General characteristics of the subjects

\begin{tabular}{lc}
\hline Measurement & Pre-test \\
\hline Age (years) & $24.1 \pm 4.2$ \\
Gender (male/female) & $9(40 \%) / 11(60 \%)$ \\
Height $(\mathrm{cm})$ & $168.6 \pm 7.7$ \\
Weight $(\mathrm{kg})$ & $59.6 \pm 9.8$ \\
\hline
\end{tabular}

have focused on effects of the exercise on the muscles of the scapulae and the trunk based on movements of other body segments ${ }^{9}$. In this context, we investigated the muscle activities of the upper trapezius, serratus anterior muscle, the lifted external oblique, and the opposite internal oblique when the lifted leg is maintained against the resistance to the directions of abduction, adduction, extension, and flexion during knee push up plus (KPP).

\section{SUBJECTS AND METHODS}

Twenty healthy subjects ( 9 males, 11 females) volunteered to participate in this study. All subjects were right-side dominant and met the requirements for muscle power, range of motion, and balance ability for the study (Table1). Winging scapula or neck and shoulder pain subjects was excluded. This study was conducted in accordance with the Declaration of Helsinki. The subjects provided informed consent prior to their participation.

Muscle activity of the upper trapezius (UT), sarratus anterior (SA), homolateral external oblique (EO), and heterolateral internal oblique (IO) muscles were recorded using a MP150WSW electromyography system (BIOPAC Systems, Santa Barbara, CA, USA) and surface EMG bipolar electrodes $20 \mathrm{~mm}$ in diameter. All EMG signals were amplified, bandpass filtered $(30-450 \mathrm{~Hz})$, and sampled rate of $1 \mathrm{kHz}$. UT electrodes were placed on the muscle belly midway between the C7 spinous process and the trapezius insertion, at the right acromioclavicular joint. SA electrodes were placed on the muscle belly at the mid-axillary line of the right (dominant) side, over the fifth rib. EO electrodes were placed parallel to the muscle fibers approximately $15 \mathrm{~cm}$ lateral to the umbilicus. IO electrodes were placed midway between the anterior iliac spine and symphysis pubis, above the inguinal ligament ${ }^{10)}$. To normalize the EMG data, maximum voluntary isometric contractions of the above mentioned muscles conducted, and a mean value of the EMG data for all tasks was expressed as a percentage relative to maximum voluntary isometric contractions.

A subject lifted a dominent leg during the KPP, maintaining the posture while the lifted leg was against the four resistance direction including abduction, adduction, extension, and flexion. In the four-point kneeling position, the subjects put their arms and legs at the shoulder width while their shoulder, hip, and knee joints were flexed at 90 degrees. The head, the spine, the pelvis, and the lifted leg were in a straight line for natural state, while the subjects were asked to see a spot on the floor. Their elbows were completely stretched and their ankle joints were in plantar flexion. As for the resistance to the legs during the exercise, a $5 \mathrm{~kg}$ weight and $3 \mathrm{~m}$ rope were put to the ankles using a sling hanging device. All the exercises were repeatedly measured three times with a five-second rest. A three-minute rest was provided between each experiment. To reduce variability, one investigator handled the electrodes in all the experiments, and the order of the exercises was randomly applied. In this study, the four methods of exercises were as follows: Exercise 1 indicates the lifted leg maintains the posture against the resistance to abduction; Exercise 2 means against the resistance to adduction; Exercise 3 indicates the resistance to extension; and Exercise 4 means the resistance to flexion.

The SPSS 17.0 statistical package was used for statistical analysis. Differences in four muscles activities during KPP with the dominant right leg extended on the mat, with various resistance condition on the extended leg were tested by repeated one-way ANOVA. The major effects were evaluated using Bonferroni"s correction, and results were considered significant at $\mathrm{p}<0.05$.

\section{RESULTS}

The muscle activity of the serratus anterior and external oblique showed the most significant increase when the subjects maintained the lifted leg against extension during the KPP $(\mathrm{p}<0.05)$. The muscle activity of the internal oblique showed the most significantly highest value when the lifted leg was maintained against adduction during the KPP (p<0.05) (Table 2).

\section{DISCUSSION}

This study was to compared the muscle activity of the trunk muscles based on the various resistance directions in the lower limbs while lifting a leg in KPP. The muscle activity of the serratus anterior and external oblique muscles significantly increased when the lifted leg was maintained against extension during the KPP. This is because the general extension torque in the lumbar spine increases by the force of the gluteus maximus, which tightens the thoracolumbar fascia and makes the 
Table 2. Comparison of EMG data in 4 knee push-up-plus exercise

\begin{tabular}{lrlll}
\hline Muscle & Exercise 1 & Exercise 2 & Exercise 3 & Exercise 4 \\
\hline Upper trapezius & $12.9 \pm 13.6$ & $13.3 \pm 13.2$ & $13.6 \pm 11.9$ & $9.39 \pm 5.8$ \\
Serratus anterior & $42.76 \pm 18.2$ & $53.3 \pm 24.7$ & $64.3 \pm 28.6^{*}$ & $43.0 \pm 21.8$ \\
External oblique & $48.5 \pm 21.3$ & $48.5 \pm 18.9$ & $49.7 \pm 21.1^{*}$ & $38.3 \pm 18.1$ \\
Internal oblique & $36.3 \pm 19.3$ & $52.0 \pm 6.2^{*}$ & $42.1 \pm 25.4$ & $30.5 \pm 13.7$ \\
\hline
\end{tabular}

$* \mathrm{p}<0.05$

force delivered to the external oblique by fascia connection, and the force finally affects the serratus anterior muscle ${ }^{11,12)}$. Meanwhile, such increase was caused by the increased recruitment of the muscular fibers in the serratus anterior muscle for the lifted leg which were induced by activation of the opposite internal oblique and the external oblique for the lifted leg due to fascia connection, an increase that was caused by the enhanced activities of the stabilizing muscles around the hip joints increased due to the opposite leg applied by weight bearing in order to maintain the posture ${ }^{12)}$. Finally the muscle activity of the internal oblique significantly increased when the lifted leg was maintained against the resistance to adduction during the push-up plus. Such increase might be explained by a theory of deep frontline in the fascia connection ${ }^{12)}$. When a leg is adducted against resistance, the great adductor muscles are moved, whose fascia is connected to the periosteum of the sciatic tuber. The force from the great adductor muscles passes the fascia of the obturator muscle connected to the periosteum of the sciatic tuber, heading for the levator ani muscle and the pelvic floor muscle. Conclusively, contraction of the pelvic floor muscle may induce contraction of the deep muscles, significantly increasing the muscle activity of the internal oblique ${ }^{12,13)}$. The results of this study may suggest that appropriate resistance directions and postures should be considered in order to selectively strengthen muscles in exercises for stabilizing the scapulae because the muscle activities in the shoulder and trunk muscles can vary based on the resistance directions on the lifted leg during the KPP. In addition, KPP mainly used for stabilizing the scapulae may have positive influence on stabilization of trunk muscles such as the internal and the external oblique based on postures and methods of exercise.

There are some limitations in this study. First, we could not identify changes in the opposite because we measured the muscles in the right shoulder joints, a side that was dominant for the subjects. It was insufficient to assess the position of the scapulae of the subjects on an object basis.

\section{REFERENCES}

1) Cools AM, Declercq GA, Cambier DC, et al.: Trapezius activity and intramuscular balance during isokinetic exercise in overhead athletes with impingement symptoms. Scand J Med Sci Sports, 2007, 17: 25-33. [Medline]

2) Kim B, Gong W, Lee S: The effect of push-up plus exercise with visual biofeedback on the activity of shoulder stabilizer muscles for winged scapula. J Phys Ther Sci, 2010, 22: 355-358. [CrossRef]

3) Ludewig PM, Cook TM: Alterations in shoulder kinematics and associated muscle activity in people with symptoms of shoulder impingement. Phys Ther, 2000, 80: 276-291. [Medline]

4) Mottram SL: Dynamic stability of the scapula. Man Ther, 1997, 2: 123-131. [Medline] [CrossRef]

5) Ellenbecker TS, Davies GJ: Closed kinetic chain exercise: a comprehensive guide to multiple joint exercise. Human Kinetics, 2001.

6) McMullen J, Uhl TL: A kinetic chain approach for shoulder rehabilitation. J Athl Train, 2000, 35: 329-337. [Medline]

7) Yang J, Lee J, Lee B, et al.: The effects of active scapular protraction on the muscle activation and function of the upper extremity. J Phys Ther Sci, 2014, 26: 599-603. [Medline] [CrossRef]

8) Batbayar Y, Uga D, Nakazawa R, et al.: Effect of various hand position widths on scapular stabilizing muscles during the push-up plus exercise in healthy people. J Phys Ther Sci, 2015, 27: 2573-2576. [Medline] [CrossRef]

9) Maenhout A, Van Praet K, Pizzi L, et al.: Electromyographic analysis of knee push up plus variations: what is the influence of the kinetic chain on scapular muscle activity? Br J Sports Med, 2010, 44: 1010-1015. [Medline] [CrossRef]

10) Criswell E: Cram's introduction to surface electromyography. Jones \& Bartlett Publishers, 2010.

11) Stevens VK, Vleeming A, Bouche KG, et al.: Electromyographic activity of trunk and hip muscles during stabilization exercises in four-point kneeling in healthy volunteers. Eur Spine J, 2007, 16: 711-718. [Medline] [CrossRef]

12) Trains A: Myofascial meridians for manual and movement therapists. Thomas W. Myers, 2009.

13) Porterfield JA, DeRosa CP: Mechanical shoulder disorders. Elsevier, 2003. 